\title{
Prevalence of metabolic syndrome in women with polycystic ovary syndrome attending a tertiary care hospital in West Delhi, India
}

\author{
Poonam Laul$^{1}$, Urvashi Miglani ${ }^{1 *}$, Anish Laul², Pinkee Saxena ${ }^{1}$, \\ Megha Malhotra ${ }^{1}$, V. K. Kadam ${ }^{1}$
}

${ }^{1}$ Department of Obstetrics and Gynecology, Deen Dayal Upadhay Hospital, New Delhi, India

${ }^{2}$ Department of Obstetrics and Gynecology, Maulana Azad Medical College, New Delhi, India

Received: 30 October 2018

Accepted: 07 January 2019

*Correspondence:

Dr. Urvashi Miglani,

E-mail: urvashim513@gmail.com

Copyright: () the author(s), publisher and licensee Medip Academy. This is an open-access article distributed under the terms of the Creative Commons Attribution Non-Commercial License, which permits unrestricted non-commercial use, distribution, and reproduction in any medium, provided the original work is properly cited.

\begin{abstract}
Background: The aim of the present study was to evaluate the prevalence of metabolic syndrome in women with polycystic ovary syndrome (PCOS).

Methods: All the women attending the gynae out-patient department of our hospital were screened for polycystic ovary syndrome as diagnosed by the Rotterdam ESHRE/ASRM-sponsored PCOS consensus workshop group, 2004. Two hundred women with PCOS underwent screening for metabolic syndrome as defined by the national cholesterol education program adult treatment panel III (ATPIII) (2001) definition and the prevalence of metabolic syndrome was compared with two hundred age and BMI matched healthy control subjects. A multivariate logistic regression analysis was applied, and significant predictors identified for the prediction of metabolic syndrome.

Results: The prevalence of metabolic syndrome among PCOS patients was $42 \%$ in present study group as compared to $14 \%$ in control group $(\mathrm{p}=0.01)$. The prevalence of metabolic syndrome was even higher in obese PCOS Vs non obese PCOS (52\% Vs $28.6 \%$ ). But even non-obese PCOS had higher prevalence of metabolic syndrome as compared to controls (28.6\% Vs $14 \%$ ).

Conclusions: The study suggests a high prevalence of metabolic syndrome in patients with PCOS \& thus it is important to screen all PCOS patients for manifestations of metabolic syndrome \& its cardiovascular sequelae.
\end{abstract}

Keywords: Metabolic syndrome, Polycystic ovary syndrome, Screening

\section{INTRODUCTION}

Polycystic ovary syndrome (PCOS) is one of the most common endocrine disorders in women, affecting approximately $5 \%$ to $10 \%$ of premenopausal women. ${ }^{1}$ The 2003 Rotterdam consensus workshop of the European society for human reproduction and embryology and the American society for reproductive medicine (ESHRE/ASRM) proposed a definition of PCOS with at least two of the following three criteria: oligomenorrhea/amenorrhea $(\mathrm{O})$, clinical or biochemical hyperandrogenism (HA), and polycystic ovaries (PCO) on ultrasonography. ${ }^{2}$ Insulin resistance is one of the key components in the pathophysiology of PCOS. ${ }^{3}$ This association clinically ramifies in the form of increased prevalence of metabolic syndrome in PCOS. Metabolic syndrome is a cluster of cardiovascular risk factors that are associated with glucose intolerance, dyslipidaemia, obesity, and hypertension. ${ }^{4,5}$ This illustrates the importance of early detection of insulin resistance and metabolic syndrome with subsequent application of preventive measures in women with polycystic ovary syndrome. 
The original national cholesterol education programmeadult treatment panel III (NCEP-ATP111) criteria in 2001 defines metabolic syndrome as the co-occurrence of three or more of the following risk factors: central obesity with waist circumference $\geq 88 \mathrm{~cm}$ in women, elevated systolic and/or diastolic blood pressure of $\geq 130 / 85$ $\mathrm{mmHg}$, impaired fasting serum glucose $\geq 110 \mathrm{mg} / \mathrm{dL}$, elevated fasting serum triglycerides $\geq 150 \mathrm{mg} / \mathrm{dL}$, fasting high-density lipoprotein (HDL) cholesterol $<50 \mathrm{mg} / \mathrm{dL}^{6}$

For Asian populations, the cut-off value for waist circumference was $\geq 90 \mathrm{~cm}$ in men and $\geq 80 \mathrm{~cm}$ in women based on the international diabetes foundation (IDF). ${ }^{7}$

The prevalence of Met S in women with PCOS has been reported to be $43.0-46.0 \%$ in the United States and 28.4 $\%$ in Brazil. ${ }^{8,9}$ In India the prevalence varies from 36 to $37.5 \%$. These varied data indicate the need for evaluation of metabolic syndrome in different populations, as it would help in planning screening strategies to prevent long-term effects. Hence, the present study was planned to estimate the prevalence of metabolic syndrome in women with PCOS presenting in our hospital and to compare it with age and BMI matched healthy control subjects.

\section{METHODS}

Patients attending the out-patient department of Deen Dayal Upadhaya hospital were screened for polycystic ovary syndrome using the Rotterdam ESHRE/ASRMsponsored PCOS consensus workshop group, 2004. ${ }^{2}$

PCOS was diagnosed if at least two of the following three features are present: oligomenorrhea/amenorrhea, clinical and /or biochemical evidence of hyperandrogenism. Polycystic ovaries on ultrasonography: enlarged ovaries $>10 \mathrm{~cm}^{3}$ on USG. Multiple peripheral ovarian follicles 810 in number and $2-8 \mathrm{~mm}$ in diameter.

\section{Exclusion criteria}

- Patients with history of diabetes mellitus

- History of drug intake such as glucocorticoids, oral contraceptive pills within three months of enrolment

- Androgen secreting tumor

- Presence of endocrinopathies causing amenorrhea such as thyroid disorders and pituitary insufficiency, Cushing syndrome

The study was approved by the clinical research ethics committee of the DDU hospital and written informed consent was obtained from all study subjects. A detailed clinical history including menstrual history, past history and family history was obtained. A complete clinical examination was done. Serum TSH, serum prolactin, Day $2 \mathrm{FSH}$ and $\mathrm{LH}$ and Serum free testosterone was done besides the routine investigations. Metabolic syndrome was diagnosed using criteria of national cholesterol education program adult treatment panel III (ATPIII). ${ }^{6}$

\section{Statistical analysis}

Statistical analysis was done using SPSS version 15.0 (SPSS, Inc., Chicago, IL, US). Continuous variables were summarized as mean with standard deviation and analyzed using sample t-test. Categorical variables were expressed as proportions and analyzed by Chi-square test.

Univariate analysis was applied to quantify the association between clinical and laboratory variables and the presence of metabolic syndrome. Multivariate logistic regression analysis was used to examine independent predictors of metabolic syndrome and to adjust for confounding factors. A $\mathrm{P}$ value of less than 0.05 was considered statistically significant.

\section{RESULTS}

The subjects ranged in age from 14-36 years; the range in body mass index (BMI) was $19-35 \mathrm{~kg} / \mathrm{m}^{2}$. A 200 patients with PCOS were recruited in present study and out of these, $84(42 \%)$ met criteria for the metabolic syndrome as diagnosed by NCEP III criteria.

In the control group comprising of 200 age and BMI matched healthy volunteers, 28 (14\%) met criteria for the metabolic syndrome $(\mathrm{p}=0.01)$.

The prevalence of the metabolic syndrome did not differ significantly between different racial/ethnic groups in both patient and control arms in present study.

Table 1: Clinical characteristics of the two groups.

\begin{tabular}{|l|l|l|l|}
\hline & $\begin{array}{l}\text { PCOS } \\
\text { group }\end{array}$ & $\begin{array}{l}\text { Control } \\
\text { group }\end{array}$ & P value \\
\hline Age (years.) & $25.1 \pm 6.3$ & $25.5 \pm 5.2$ & $\mathrm{P}=\mathrm{NS}$ \\
\hline BMI $\left(\mathrm{kg} / \mathrm{m}^{2}\right)$ & $27.2 \pm 0.3$ & $26.9 \pm 0.4$ & $\mathrm{P}=\mathrm{NS}$ \\
\hline $\begin{array}{l}\text { Waist } \\
\text { circumference }(\mathrm{cm})\end{array}$ & $95 \pm 6.4$ & $84 \pm 5.6$ & $\mathrm{P}=0.01$ \\
\hline SBP $(\mathrm{mmHg})$ & $126 \pm 5$ & $116 \pm 3.1$ & $\mathrm{P}=0.01$ \\
\hline DBP $(\mathrm{mmHg})$ & $83 \pm 3.2$ & $76 \pm 3.9$ & $\mathrm{P}=0.01$ \\
\hline
\end{tabular}

As shown in Table 1, the two groups were similar in age (25.1 \pm 6.3 vs. $25.5 \pm 5.2$ years.) and BMI (27.2 \pm 0.3 vs. $26.9 \pm 0.4)$. However other characteristics of metabolic syndrome were significantly higher in the PCOS group as compared to control group.

Table 2: Biochemical characteristics of the two groups.

\begin{tabular}{|l|l|l|l|}
\hline & $\begin{array}{l}\text { PCOS } \\
\text { group }\end{array}$ & $\begin{array}{l}\text { Control } \\
\text { group }\end{array}$ & $\begin{array}{l}\text { P } \\
\text { value }\end{array}$ \\
\hline $\begin{array}{l}\text { Blood sugar fasting } \\
\text { (mg/dl) }\end{array}$ & $94 \pm 12$ & $82 \pm 10$ & $\mathrm{P}=0.05$ \\
\hline Triglycerides (mg/dl) & $156 \pm 45$ & $135 \pm 25$ & $\mathrm{P}=0.03$ \\
\hline HDL (mg/dl) & $46 \pm 5$ & $51 \pm 7$ & $\mathrm{P}=0.05$ \\
\hline LDL (mg/dl) & $110 \pm 13$ & $100 \pm 12$ & $\mathrm{P}=0.10$ \\
\hline
\end{tabular}


Fasting glucose $(94 \pm 12$ vs $82 \pm 10 \mathrm{mg} / \mathrm{dl})$ levels were not statistically significantly different between the two groups.

Table 3: Prevalence of the metabolic syndrome according to age group.

\begin{tabular}{|l|l|l|l|}
\hline Years & PCOS group & Control group & P value \\
\hline $11-20$ & $2 / 11(18 \%)$ & $0 / 9(0 \%)$ & $\mathrm{p}=0.001$ \\
\hline $21-30$ & $14 / 30(46.6 \%)$ & $4 / 31(12.9 \%)$ & $\mathrm{p}=0.005$ \\
\hline $31-40$ & $5 / 9(55.5 \%)$ & $3 / 10(30 \%)$ & $\mathrm{P}=0.006$ \\
\hline
\end{tabular}

However, triglycerides were higher in PCOS group as compared to control group (Table 2).

When PCOS patients were stratified according to age group, it was found that prevalence of metabolic syndrome was significantly higher with increasing age (55.5\% in 31-40 years Vs $18 \%$ in $11-20$ years; $p=0.001)$. This pattern was observed in both PCOS and control groups, however prevalence of metabolic syndrome was higher in all age stratified PCOS groups as compared to controls (Table 3).

Table 4: Comparison of PCOS women with and without the metabolic syndrome (clinical parameters).

\begin{tabular}{|l|l|l|l|}
\hline & With metabolic syndrome $(\mathbf{n = 2 1})$ & Without metabolic syndrome $(\mathbf{n = 2 9})$ & P value \\
\hline Age $($ years.) & $27 \pm 3.1$ & $23 \pm 2.6$ & $P=0.05$ \\
\hline BMI $\left(\mathrm{kg} / \mathrm{m}^{2}\right)$ & $29.4 \pm 0.8$ & $26.6 \pm 1.2$ & $\mathrm{P}=0.04$ \\
\hline Waist circumference $(\mathrm{cm})$ & $100 \pm 1.5$ & $90 \pm 1.2$ & $\mathrm{P}=0.01$ \\
\hline SBP $(\mathrm{mmHg})$ & $130 \pm 4.2$ & $120 \pm 3.9$ & $\mathrm{P}=0.05$ \\
\hline DBP $(\mathrm{mmHg})$ & $84 \pm 3.4$ & $80 \pm 4.5$ & $\mathrm{P}=0.04$ \\
\hline
\end{tabular}

Table 5: Comparison of PCOS women with and without the metabolic syndrome (biochemical parameters).

\begin{tabular}{|l|l|l|l|}
\hline & With metabolic syndrome (42\%) & Without metabolic syndrome (58\%) & P value \\
\hline Blood sugar fasting $(\mathrm{mg} \%)$ & $96 \pm 3.8$ & $92 \pm 4.2$ & $\mathrm{P}=0.06$ \\
\hline Triglycerides $(\mathrm{mg} / \mathrm{dl})$ & $175 \pm 15$ & $141 \pm 14$ & $\mathrm{P}=0.02$ \\
\hline HDL $(\mathrm{mg} / \mathrm{dl})$ & $40 \pm 5$ & $51 \pm 3.6$ & $\mathrm{P}=0.02$ \\
\hline LDL $(\mathrm{mg} / \mathrm{dl})$ & $112 \pm 9$ & $109 \pm 6.4$ & $\mathrm{P}=\mathrm{NS}$ \\
\hline
\end{tabular}

As expected, compared with women who did not meet criteria for the metabolic syndrome, those with the metabolic syndrome had a significantly higher BMI $\left(29.4 \pm 0.8\right.$ Vs. $\left.26.6 \pm 1.2 \mathrm{~kg} / \mathrm{m}^{2}\right)$, waist circumference $(100 \pm 1.5$ Vs. $90 \pm 1.2 \mathrm{~cm})$, systolic $(130 \pm 4.2$ Vs. $120 \pm 3.9$ $\mathrm{mm} \mathrm{Hg})$ and diastolic $(84 \pm 3.4$ Vs. $80 \pm 4.5 \mathrm{~mm} \mathrm{Hg})$ blood pressures. PCOS women with metabolic syndrome were significantly older, had more central obesity and had higher systolic and diastolic BP (Table 4).

Table 6: Prevalence of individual components of the metabolic syndrome.

\begin{tabular}{|l|l|l|l|}
\hline & $\begin{array}{l}\text { PCOS } \\
\text { group (\%) }\end{array}$ & $\begin{array}{l}\text { Control } \\
\text { groujp }(\%)\end{array}$ & P value \\
\hline $\begin{array}{l}\text { Waist circumference } \\
>88 \mathrm{~cm}\end{array}$ & 78 & 20 & $\mathrm{P}=0.001$ \\
\hline $\begin{array}{l}\text { Triglycerides } \\
>150 \mathrm{mg} / \mathrm{dl}\end{array}$ & 52 & 14 & $\mathrm{P}=0.002$ \\
\hline HDL <50mg/dl & 46 & 18 & $\mathrm{P}=0.002$ \\
\hline $\begin{array}{l}\text { SBP/DBP } \\
>130 / 85 \mathrm{~mm} \mathrm{Hg}\end{array}$ & 32 & 12 & $\mathrm{P}=0.001$ \\
\hline FBS $>110 \mathrm{mg} / \mathrm{dl}$ & 6 & 2 & $\mathrm{P}=0.01$ \\
\hline
\end{tabular}

When individual components of metabolic syndrome were compared in PCOS and controls, it was found that all components of metabolic syndrome were significantly higher in PCOS group as compared to controls. However, fasting blood glucose was increased in only few patients $(6 \%)$. When PCOS patients were stratified according to BMI as obese Vs non-obese PCOS, it was found that prevalence of metabolic syndrome was significantly higher in obese PCOS (52\% vs. $28.6 \%$; $=0.05$ ). This pattern was similar among all the individual components of metabolic syndrome. However, even among non-obese PCOS group, prevalence was significantly higher than control group $(28.6 \%$ vs $14 \%, \mathrm{p}=0.05)$, signifying that factors other than obesity also contribute to increased prevalence of metabolic syndrome among PCOS patients.

\section{DISCUSSION}

The prevalence of metabolic syndrome among patients with PCOS was found to be $42 \%$ in present study. This is significantly higher than the prevalence of metabolic syndrome in age and BMI matched control group (14\%, $\mathrm{p}=0.01)$. This data suggests a very high prevalence of metabolic syndrome in patients with PCOS, with its inherent cardiovascular risks. Therefore, it is imperative to screen every PCOS patient for metabolic syndrome to predict and prevent cardiovascular complications. Shroff et al, have demonstrated that young, obese women with PCOS have a high prevalence of early asymptomatic 
coronary atherosclerosis, compared with obese controls. ${ }^{10}$ This increased risk is independent of traditional cardiovascular risk factors and novel markers of inflammation. These findings reiterate the need to screen, counsel and treat these women to prevent symptomatic cardiovascular disease.

Present findings are in accordance with findings of Glueck et al, who found a very high prevalence of metabolic syndrome (46\%) in their study on 138 oligoamenorrheic white women with PCOS. ${ }^{11}$ After age adjustment, $46.4 \pm 4.2 \%$ of women with PCOS had the metabolic syndrome versus $22.8 \pm 1.1 \%$ of control women $(p<0001)$. They further found that metformin and diet ameliorated many of the features of the metabolic syndrome in these patients thereby reducing risk for atherothrombosis and type 2 diabetes mellitus in these patients.

However, Carmina et al, in contrast have found a much lower prevalence $(8.2 \%)$ of metabolic syndrome in Italian women with PCOS probably due to lower prevalence of obesity and hypertriglyceridemia in Italian women as well as differences in ethnicity and dietary patterns. ${ }^{12}$

When patients in present study were stratified according to age, the prevalence of metabolic syndrome increased steadily with each decade $(18 \%, 46 \%$ and $55 \%$ respectively in 11-20years, 21-30years and 31-40 years), as has been observed in previous studies.

This pattern was observed in the control group also. Also, this analysis was significant because of the finding that more than one third of non-diabetic women with PCOS have developed the metabolic syndrome well before the end of their fourth decade, and usually before the end of their third decade of life.

This prevalence is markedly higher than the $6.7 \%$ prevalence of metabolic syndrome reported in women between the ages of 20 and 30 years and the $15 \%$ prevalence reported in women between ages 30 and 40 years from the third national health and nutrition examination survey (NHANES III). Ehrmann et al, found that the risk of having metabolic syndrome in the older group (>35 years) was nine times higher than that in the younger group $(20-24$ years, $\mathrm{p}=0.0001) .{ }^{13}$

The prevalence of metabolic syndrome was significantly higher in obese PCOS in the present study. Similar findings were reported by Kavitha et al, who found that the infertile women with PCOS, particularly those with age $\geq 25$ years or with central obesity (a waist hip ratio of $\geq 0.85$ ), are at a higher risk of developing metabolic syndrome. ${ }^{14}$ Therefore, it is recommended that all women with PCOS especially, older and obese PCOS should be screened for the presence of metabolic syndrome.

However, even among non-obese PCOS group, prevalence was significantly higher than control group
(28.6\% vs. $14 \%, \mathrm{p}=0.05)$, signifying that factors other than obesity also contribute to increased prevalence of metabolic syndrome among PCOS patients. It is important to note that there is substantial variation in the prevalence of metabolic syndrome in PCOS, depending upon the population studied and criteria used to define the PCOS and metabolic syndrome

For example, using national cholesterol education program III criteria 6, the metabolic syndrome was evident in only $1.6 \%$ of Czech women with PCOS, which was not significantly different from that observed in controls. ${ }^{15}$ On the other hand, a study on 837 Korean women found the prevalence of MS to be more than $30 \%$, the incidence being higher in non-obese PCOS women with hyperandrogenism and menstrual irregularity. ${ }^{16}$ Zhao et al, analyzed that East Asian women with PCOS have a lower BMI and a milder hyper androgenic phenotype, but have the highest prevalence of metabolic syndrome. South Asians in particular have a high prevalence of insulin resistance and metabolic syndrome, and are at risk for type 2 diabetes, with central obesity more than BMI reflecting their metabolic risk. African American and Hispanic women are more obese and more prone to metabolic problems. He concluded that ethnically appropriate guidelines are needed for identifying anthropometric thresholds for better screening and diagnosis in high-risk ethnic groups. ${ }^{17}$

Authors also sought to determine the prevalence of individual components of metabolic syndrome among patients with PCOS. Our observations suggest a high prevalence of all the individual components of metabolic syndromes in PCOS patients as compared to age and BMI matched controls. Increased waist circumference was the most prevalent feature of metabolic syndrome (78\%). However, only few patients had increased fasting blood glucose $>110 \mathrm{mg} / \mathrm{dl}(6 \%)$. Patients who were proved to be diabetic upon oral glucose tolerance testing were excluded from this analysis to avoid the confounding effects of the diabetic state on measures that define the metabolic syndrome.

Waist circumference below the threshold of $88 \mathrm{~cm}$ was a relatively rare occurrence but was highly predictive of the absence of the metabolic syndrome. Conversely, an elevated fasting glucose concentration was highly predictive of the metabolic syndrome, but its practical utility appears to be diminished by the fact that only $6 \%$ of subjects had this finding.

Previous studies have examined whether factors known to enhance risk for type 2 diabetes also influenced the risk for metabolic syndrome in PCOS. In one study, one third of women with the metabolic syndrome had a first degree-relative with type 2 diabetes, whereas $26 \%$ of those without the metabolic syndrome had a diabetic first-degree relative, a difference that was not significant. ${ }^{18}$ Of note, however, was the finding that women with a family history of diabetes had a 
significantly greater number of individual components of the metabolic syndrome.

The prevalence of metabolic syndrome in PCOS was 3.46.6 times higher than in the matched control population suggesting that PCOS per se results in a higher prevalence of metabolic syndrome. Further, our data also suggest that the prevalence of metabolic syndrome is 3 times higher than in the control group. Also, the prevalence of metabolic syndrome in PCOS is strongly influenced by the criteria used to diagnose metabolic syndrome as well as the criteria used to diagnose PCOS. However, it seems clear that metabolic syndrome is much more common in PCOS than in the general population in women of similar age.

In summary, present study reports a very high prevalence of metabolic syndrome (42\%) in Indian urban population of PCOS patients as compared to age and BMI matched controls. Prevalence in obese PCOS patients was even higher. Even among non-obese PCOS, prevalence was much higher than controls (28.6\% Vs $14 \%$ ). This data has important implications for gynecologists caring for these patients and it is extremely important to screen all patients with PCOS for metabolic syndrome and its attendant cardiovascular risks.

The metabolic syndrome is evident at an early age in women with PCOS, irrespective of race and ethnicity. Hyperinsulinemia, a central factor in the pathogenesis of PCOS, also appears to be a critical link between PCOS and the metabolic syndrome. Strategies designed to attenuate insulin resistance have proved to be of benefit in the treatment of both syndromes; whether such strategies will lead to a reduction in risk of developing cardiovascular disease and type 2 diabetes remains to be determined.

However, there are few limitations of present study. First the sample size is small, and it is not statistically powered for subgroup analysis. Secondly since present study was conducted at a tertiary care hospital, present study group comprised of patients who had more advanced pathophysiological manifestations of the disease and it may not be true representative of the prevalence in the community. Thirdly, our research has primarily focused on metabolic syndrome features and has not adequately examined the natural history of metabolic disease in PCOS across the different diagnostic phenotypes of women.

\section{CONCLUSION}

In conclusion, women with PCOS show an unfavourable cardiovascular risk profile, high need for screening and intervention at a young age to avoid significant morbidity. The gynaecologist could therefore play an important role in identification of women at risk. Ultimately, classification of the metabolic complications for each phenotype will provide an evidence base for screening of metabolic risk upon diagnosis of PCOS and may guide optimal treatment to prevent metabolic complications of PCOS. This will also assist in understanding the underlying pathophysiology of metabolic features of this condition.

Funding: No funding sources

Conflict of interest: None declared

Ethical approval: The study was approved by the Institutional Ethics Committee

\section{REFERENCES}

1. Romero R, Kalache KD, Kada N. Timing the delivery of the preterm severely growth restricted fetus: venous Doppler, cardiotocography on the biophysical profile? Ultrasound Obstet Gynacol. 2002;19:118-21.

2. Giles WB, Trudringer BJ, Baird PJ. Fetal Umbilical flow velocity wave form and placental resistance pathological co-relation. $\mathrm{Br} \mathrm{J}$ Obstet Gynacol. 1985;92:31-8.

3. Mendez MA, Gayta MV, Flores R. Doppler ultrasound evaluation in preeclampsia. BMC Res Notes. 2013;19:477.

4. Gramellini D, Folli MC, Raboni S, Vadora E, Merialdi A. Cerebral-umbilical Doppler ratio as a predictor of adverse perinatal outcome. Obstet Gynecol. 1992;79(3):416-20.

5. Bano S, Chaudhary V, Pande S, Mehta VC, Sharma AK. Colour Doppler evaluation of cerebral umbilical pubatility ratio and its usefulness in the diagnosis of intrauterine growth restriction and prediction of adverse perinatal outcome. Indian J Radiol Imaging. 2010;20(1):20-5

6. Mari G, Hanif F, Kruger M, Cosmi E, Forgas SJ, Treadwell MC. Middle cerebral artery peak systolic velocity a new Doppler parameter in the assessment of growth restricted fetus. Ultrasound Obstet Gynacol. 2007;29(3):310-6.

7. Schenone MH, Mari G. The MCA Doppler and its role in the evaluation of fetal anemia and fetal growth restriction. Clin Perinatal.2011;38(1):83-102.

8. Hecher K, Campbell S, Doyle P, Harrington K, Nicoladies K. Asessment of fetal compromise by Doppler ultrasound investigation of the fetal circulation. Circulation. 1995;91:129-38.

9. Baschat AA, Gembruch U, Weiner CP, Harman CR. Qualitative venous Doppler waveforms analysis improves prediction of critical perinatal outcome in premature growth restricted foetuses. Ultrasound Obstet Gynacol. 2003;22:240-5.

10. Brown MA, Lindheimer MD, Swiet M, Assche VA, Moutquin JM. The classification and diagnosis of the hypertensive disorders of pregnancy: statement from the international society for the study of hypertension in pregnancy (ISSHP). Hypertens Pregnancy. 2001;20:19-24

11. Kurmanavicius J, Florio I, Wisser J, Hebisch G, Zimmermann R, Muller R et al. Refence resistance 
indices of the umbilical, fetal middle cerebral and uterine arteries at 24-42 weeks of gestation. Ultrasound Obstet Gynecol. 1997;10:112-20.

12. Ozeren M, Dinc H, Ekmen U, Senekayli C, Aydemir V. Umbilical and middle cerebral artery Doppler indices in patients with preeclampsia. Eur J Obstet Gynecol Reprod Biol. 1999;82:11-6.

13. Yoon BH, Lee CM, Kim SW. An abnormal umbilical artery waveform: A strong and independent predictor of adverse perinatal outcome in patients with preeclampsia. Am J Obs Gyn. 1994;171:713-21.

Cite this article as: Laul P, Miglani U, Laul A, Saxena P, Malhotra M, Kadam VK. Prevalence of metabolic syndrome in women with polycystic ovary syndrome attending a tertiary care hospital in West Delhi, India. Int J Reprod Contracept Obstet Gynecol 2019;8:1127-32. 